\title{
Absorption and eigenmode calculation for one-dimensional periodic metallic structures using the hydrodynamic approximation
}

Yanai, Avner; Mortensen, N. Asger; Levy, Uriel

Published in:

Physical Review B Condensed Matter

Link to article, DOI:

10.1103/PhysRevB.88.205120

Publication date:

2013

Document Version

Publisher's PDF, also known as Version of record

Link back to DTU Orbit

Citation (APA):

Yanai, A., Mortensen, N. A., \& Levy, U. (2013). Absorption and eigenmode calculation for one-dimensional periodic metallic structures using the hydrodynamic approximation. Physical Review B Condensed Matter, 88(20), [205120 ]. https://doi.org/10.1103/PhysRevB.88.205120

\section{General rights}

Copyright and moral rights for the publications made accessible in the public portal are retained by the authors and/or other copyright owners and it is a condition of accessing publications that users recognise and abide by the legal requirements associated with these rights.

- Users may download and print one copy of any publication from the public portal for the purpose of private study or research.

- You may not further distribute the material or use it for any profit-making activity or commercial gain

- You may freely distribute the URL identifying the publication in the public portal 


\title{
Absorption and eigenmode calculation for one-dimensional periodic metallic structures using the hydrodynamic approximation
}

\author{
Avner Yanai, ${ }^{1}$ N. Asger Mortensen, ${ }^{2}$ and Uriel Levy ${ }^{1, *}$ \\ ${ }^{1}$ Department of Applied Physics, The Benin School of Engineering and Computer Science, The Hebrew University of Jerusalem, Israel \\ ${ }^{2}$ DTU Fotonik, Department of Photonics Engineering, Technical University of Denmark, DK-2800 Kongens Lyngby, Denmark
}

(Received 3 July 2013; published 14 November 2013)

\begin{abstract}
We develop a modal method that solves Maxwell's equations in the presence of the linearized hydrodynamic correction. Using this approach, it is now possible to calculate the full diffraction for structures with a period of the order of the plasma wavelength, including not only the transverse but also the longitudinal modes appearing above the plasma frequency. As an example for using this method we solve the diffraction of a plane wave near the plasma frequency from a bimetallic layer, modeled as a continuous variation of the plasma frequency. We observe absorption oscillations around the plasma frequency. The lower frequency absorption peaks and dips correspond to the lowest longitudinal modes concentrated in the lower plasma frequency region. As the frequency is increased, higher order longitudinal modes are excited and extend to the region of higher plasma frequency. Moreover, an examination of the propagation constants of these modes reveals that the absorption peaks and dips are directly related to the direction of phase propagation of the longitudinal modes. Furthermore, we formulate a variant of the plane wave expansion method, and use it to calculate the dispersion diagram of such longitudinal modes in a periodically modulated plasma frequency layer.
\end{abstract}

DOI: 10.1103/PhysRevB.88.205120

PACS number(s): 42.25.Bs, 71.45.Gm, 71.45.Lr, 52.35.Fp

\section{INTRODUCTION}

Along with advances in nanoplasmonics, plasmonic devices reach length scales for which nonlocal effects of the metal electric permittivity function may no longer be neglected. For noble metals with critical dimensions in the sub-10 nm regime, the longitudinal plasmonic response exhibits spatial dispersion. This deviation from the ordinary local approximation requires modification of known analytical and numerical tools. The hydrodynamic nonlocal model ${ }^{1-4}$ can be regarded as a simple approach (compared to more complex, quantum models). However, it successfully reproduces experimental results obtained for thin layered metals ${ }^{5}$ and offers a qualitative explanation for the blueshifting of the localized surface-plasmon resonance observed in silver nanoparticles. ${ }^{6,7}$ These results cannot be explained with local models. In this paper, we study the response of a metallic layer with periodic variation of the free-carrier density, under the hydrodynamic approximation. While the hydrodynamic model fails to account for quantumsize effects, such as quantum tunneling, ${ }^{8-10}$ it is a well established model for the dimensions studied here. Until now, various numerical algorithms that solve Maxwell's equations with the hydrodynamic correction have been reported. ${ }^{11-19}$ In this paper, we provide a rigorous numerical approach that allows the calculation of one-dimensional (1D) periodic structures. Our method relies on the Fourier modal method (FMM), also known as the rigorous coupled wave analysis (RCWA) method. ${ }^{20-22}$ This method can be regarded as semianalytic in the sense that not only the field distribution is calculated, but also the propagation constants and the eigenmodes of the periodic structure are obtained, allowing to derive additional physical insight (see, e.g., Ref. 23). Adding the hydrodynamic terms to the ordinary FMM formulation allows us to utilize some of the strengths that are offered by FMM. The paper is structured as follows. In Sec. II the FMM with the additional hydrodynamic terms is presented. In addition, we formulate the band diagram dispersion calculation of the longitudinal modes. In Sec. III, results based on this framework are shown. Section IV concludes the paper.

\section{FMM WITH THE HYDRODYNAMIC CORRECTION}

First, we briefly review the essential basics of the FMM. Further details can be found in several references, e.g., Refs. 20 and 21. In its most common formulation, the FMM uses a Floquet-Bloch expansion within a unit cell $0 \leqslant x<L$ (see the schematic in Fig. 1) to represent Maxwell's equations in each $z$-invariant periodic layer. Afterwards, the eigenmodes and eigenvalues of the fields are calculated by solving an eigenvalue equation. We now elaborate on these principles. The Floquet-Bloch condition implies that the wave vectors in the $x$ direction are given by $k_{x, m}=k_{x, 0}+m K$, where $K=2 \pi / L$ is the grating vector and $k_{x, 0}$ is the "zero-order" term. With the FMM, Maxwell's equations are solved for each locally $z$-invariant layer, from an eigenvalue equation of the form $\frac{\partial}{\partial z} \mathbf{F}=\mathbf{A F}$. Here, $\mathbf{F}$ is a column vector of the Fourier components of the fields and $\mathbf{A}$ is an operator matrix defined by Maxwell's equations. The propagation constants $k_{z, n}$ are obtained by solving this eigenvalue equation. In order to solve the eigenvalue equation numerically, one must truncate the number of Fourier components to some finite number of $N$ elements, with $-\lfloor N / 2\rfloor \leqslant m \leqslant\lfloor N / 2\rfloor$. Generally, the solution converges to the exact solution by increasing $N$. In order to solve a diffraction problem, the fields in adjacent layers are matched by employing the proper boundary conditions. By this matching procedure, a mode amplitude constant $C_{n}$ is solved for the $n$th eigenmode. ${ }^{20}$ In the following we present the derivation of the matrix operator $\mathbf{A}$ in the presence of the hydrodynamic correction.

\section{A. Maxwell's equations with the hydrodynamic correction}

In each $z$-invariant layer, and for a single frequency component $\omega$, Maxwell's equations with the linearized hydrodynamic 


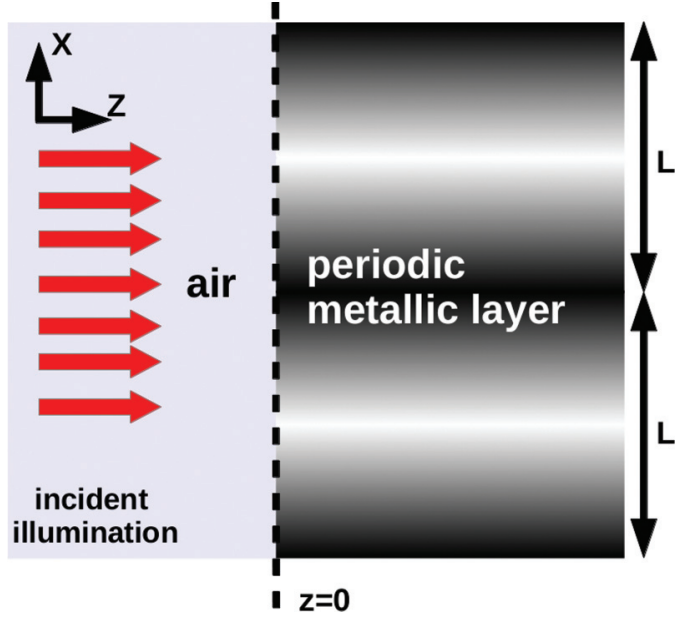

FIG. 1. (Color online) Schematic showing two unit cells of the diffraction problem geometry, with light incident from left to right. The air/metal interface is located at $z=0$, and the direction of periodicity is along the vertical (i.e., $x$ ) axis. The region with reduced free-carrier density is in the center of the unit cell (bright), whereas the boundaries of the unit cell are the region with higher free-carrier density (dark).

correction are given by ${ }^{19,24}$

$$
\begin{gathered}
\nabla \times E-j \omega \mu_{0} H=0, \\
\nabla \times H+j \omega \varepsilon_{0} E+e N_{0}(x) v=0, \\
\varepsilon_{0} \nabla \cdot E+e n_{1}=0, \\
\nabla \cdot\left(N_{0}(x) v\right)-j \omega n_{1}=0, \\
-j m \omega\left(N_{0}(x) v\right)+m \gamma\left(N_{0}(x) v\right)+N_{0}(x) e E+m \beta^{2} \nabla n_{1}=0,
\end{gathered}
$$

where $N_{0}(x)$ is a periodic function of the density of free electrons in equilibrium, $n_{1}$ is the first-order nonequilibrium correction to the equilibrium electron density, and likewise $v$ is the first-order nonequilibrium electron velocity while there are no equilibrium currents. Furthermore, the strength of the nonlocal response is governed by $\beta^{2}=\frac{3}{5} v_{F}^{2}$. The electron mass is denoted by $m$. The case where $N_{0}$ varies with $x$ while $\beta$ is constant can be regarded as a toy model for the scenario in which two metals with different plasma frequencies fill the unit cell, with continuous variation of the free-carrier density. We solve the set of Eq. (1) for TM polarization [i.e., $H(x, y, z)=\hat{y} H_{y}(x, z)$ and $\left.E(x, y, z)=\hat{x} E_{x}(x, z)+\hat{z} E_{z}(x, z)\right]$, as only this polarization supports longitudinal modes. As explained above, in order to solve Eq. (1) with standard FMM formulation, we need to isolate all $\frac{\partial}{\partial z}$ dependencies to obtain an eigenvalue equation. For convenience, we introduce $\hat{\omega}^{2}=\omega(\omega+i \gamma), \tilde{\beta}^{2}=\beta^{2} / c^{2}$, $\tilde{\omega}_{p}^{2}=\omega_{p}^{2} / c^{2}=\frac{N_{0} e^{2}}{m \varepsilon_{0}} / c^{2}, \hat{k}_{0}=\hat{\omega} / c, \tilde{\mu}_{0}=c \mu_{0}$, and $\tilde{\varepsilon}_{0}=c \varepsilon_{0}=$ $\tilde{\mu}_{0}^{-1}$. Furthermore, we define the hydrodynamic current as $J=$ $e N_{0} v$ and $J(x, y, z)=\hat{x} J_{x}(x, z)+\hat{z} J_{z}(x, z)$. Since we have spatial harmonic variations, we straightforwardly make the following substitutions for the derivatives: $\partial / \partial_{z}=j k_{0} k_{z}$ and $\partial / \partial_{x}=j k_{0} k_{x}$. Making these substitutions and performing algebraic manipulations described in some detail in Appendix A, we arrive at the eigenvalue equation in matrix form:

$$
\begin{aligned}
& {\left[\begin{array}{c}
\mathbf{E}_{\mathbf{x}} \\
\nabla \cdot \mathbf{J}
\end{array}\right]\left[\mathbf{K}_{\mathbf{z}}^{2}\right]} \\
& \quad=\left[\begin{array}{cc}
\tilde{\mu}_{0} \mathbf{I} & \mathbf{K}_{\mathbf{x}} \\
\hat{k}_{0}^{2} \tilde{\beta}^{-2} \mathbf{K}_{\mathbf{x}} & \tilde{\varepsilon}_{0} \tilde{\beta}^{-2}\left(\hat{k}_{0}^{2} \mathbf{I}-\mathbf{\Omega}_{\mathbf{p}}^{2}\right)
\end{array}\right] \\
& \quad \times\left[\begin{array}{cc}
\tilde{\varepsilon}_{0} \hat{k}_{0}^{-2}\left(\hat{k}_{0}^{2} \mathbf{I}-\mathbf{\Omega}_{\mathbf{p}}^{\mathbf{2}}\right) & -\hat{k}_{0}^{-2} \tilde{\beta}^{2} \mathbf{K}_{\mathbf{x}} \\
-\mathbf{K}_{\mathbf{x}} & \tilde{\mu}_{0} k_{0}^{-2} \mathbf{I}
\end{array}\right]\left[\begin{array}{c}
\mathbf{E}_{\mathbf{x}} \\
\nabla \cdot \mathbf{J}
\end{array}\right] .
\end{aligned}
$$

Here, $\mathbf{E}_{\mathbf{x}}$ and $\nabla \cdot \mathbf{J}$ are the eigenvector matrices of $E_{x}$ and $\nabla \cdot J$, respectively. $\mathbf{K}_{\mathbf{x}}$ and $\mathbf{K}_{\mathbf{z}}$ are diagonal matrices with elements $k_{x, m}$ and $k_{z, n}$, respectively, and the identity matrix is $\mathbf{I} . \boldsymbol{\Omega}_{\mathbf{p}}^{\mathbf{2}}$ is the Toeplitz matrix with elements corresponding to the Fourier components of $\tilde{\omega}_{p}^{2}(x)$. The matrices $\mathbf{I}, \mathbf{\Omega}_{\mathbf{p}}^{\mathbf{2}}, \mathbf{K}_{\mathbf{x}}, \mathbf{E}_{\mathbf{x}}$, and $\nabla \cdot \mathbf{J}$ are of size $N \times N$, while $\mathbf{K}_{\mathbf{z}}$ is a $2 N \times 2 N$ matrix and the overall number of eigenmodes obtained from Eq. (2) is $2 N$. However, in local media, the number of eigenmodes is equal to the truncation number, i.e., $N .^{20}$ Therefore, a total of $3 N$ mode amplitude constants need to be found when matching the fields at the interface of a local layer with a layer with nonlocal response. The ordinary boundary conditions demanding continuity of the tangential field components $E_{x}$ and $H_{y}$ provide only $2 N$ equations. To match the "missing" $N$ amplitude constants, an additional boundary condition $(\mathrm{ABC})$ is required. This is very similar to the known case of matching the field amplitudes between two homogeneous local and nonlocal layers. ${ }^{25}$ For simplicity, we consider an air/metal interface. For this case, the boundary conditions are the continuity of $E_{x}, J_{z}$, and $E_{z}$ across the interface. . $^{3,26,27} \mathrm{We}$ note, however, that the $\mathrm{ABC}$ does not change in any way the eigenvalue equation of the periodic metallic layer, which is a direct solution of Maxwell's equations with the hydrodynamic correction. In Sec. III A, we solve a full diffraction problem for the case of light incident from air on a semi-infinite periodic nonlocal layer. At the interface of both media, the mode amplitude constants are found by employing these boundary conditions. In Appendix B, we present a procedure based on the $S$-matrix algorithm ${ }^{22}$ for the calculation of the mode amplitudes. For completeness, this procedure is formulated for the more general case of a nonlocal periodic layer embedded in a local environment.

\section{B. Band diagram calculation}

In order to calculate the dispersion diagram of the longitudinal modes, we follow an approach based on that in Ref. 28. This approach is a variant of the plane wave expansion method (PWM). ${ }^{29}$ In contrast to the conventional PWM, where the Bloch wave vector $\left(k_{x, 0}\right)$ is assumed and the frequency $(\omega)$ is solved from an eigenvalue problem, in the revised PWM, the frequency is assumed beforehand, and the phase difference of the fields across the unit cell (known as the Bloch wave vector) is calculated. We note that for nondispersive lossless materials there is a freedom to choose one or the other. However, for the dispersive metal it is important that one solves for the complex Bloch wave vector while treating the frequency as real (alternatively, one can also solve for complex $\omega$; see Ref. 30). This is relevant for the experimental situation where 
the structure is probed by a cw laser with a well-defined frequency. We now show how the PWM variant can be applied to calculate the dispersion diagram of a metallic structure with the hydrodynamic correction. For simplicity, we assume a 1D case with $k_{z}=0$, and that $\omega_{p}$ is periodic with $x$. For such a case, Eq. (1) [see also Eq. (A3)] reduces to three first-order differential equations:

$$
\begin{aligned}
k_{x} E_{z}+\tilde{\mu}_{0} H_{y} & =0, \\
\tilde{\varepsilon}_{0} \varepsilon_{T} E_{z}+k_{x} H_{y} & =0, \\
E_{x}-\hat{k}_{0}^{-2} \tilde{\omega}_{p}^{2}(x) E_{x}-\hat{k}_{0}^{-2} k_{0}^{2} \tilde{\beta}^{2} k_{x}^{2} E_{x} & =0 .
\end{aligned}
$$

Here $\varepsilon_{T} \equiv 1-\frac{\tilde{\omega}_{p}^{2}(x)}{\hat{k}_{0}^{2}}$. Equations (3) can be subdivided into two independent sets: Equations (3a) and (3b) describe the transverse modes (no field components in the direction of the only nonzero $k$-vector component, i.e., $k_{x}$ ) while Eq. (3c) defines the dispersion law of the longitudinal modes. Moreover, by defining $\varepsilon_{L} \equiv 1-\frac{\tilde{\omega}_{p}^{2}(x)}{\hat{k}_{0}^{2}-k_{0}^{2} \tilde{\beta}^{2} k_{x}^{2}}$, Eq. (3c) can be rewritten as $\varepsilon_{L} E_{x}=0$, from which the familiar condition for longitudinal modes $\varepsilon_{L}=0$ is apparent. To solve Eq. (3c), we define $k_{x} \equiv k_{x, 0}+k_{x, m}$. Here $k_{0} k_{x, 0} L$ is the phase difference of the field $F$ between the two boundaries of the unit cell according to $F(x=L)=F(x=0) \exp \left(j k_{0} k_{x, 0} L\right)$ and $k_{x, m}=$ $m K$. With these definitions, and the auxiliary field quantity $\dot{E}_{x} \equiv\left[k_{x, 0}+k_{x, m}\right] E_{x}$, we split Eq. (3c) into two first-order equations

$$
\begin{gathered}
\tilde{\beta}^{-2} k_{0}^{-2}\left[\hat{k}_{0}^{2}-\tilde{\omega}_{p}^{2}(x)\right] E_{x}-\left[k_{x, 0}+k_{x, m}\right] \dot{E}_{x}=0, \\
\dot{E}_{x}=\left[k_{x, 0}+k_{x, m}\right] E_{x} .
\end{gathered}
$$

Equation (4) can be recast to the matrix form:

$$
\left[\begin{array}{c}
\dot{\mathbf{E}}_{\mathbf{x}} \\
\mathbf{E}_{\mathbf{x}}
\end{array}\right]\left[\mathbf{K}_{\mathbf{x}, \mathbf{0}}\right]=\left[\begin{array}{cc}
-\mathbf{K}_{\mathbf{x}} & \tilde{\beta}^{-2} k_{0}^{-2}\left(\hat{k}_{0}^{2} \mathbf{I}-\boldsymbol{\Omega}_{\mathbf{p}}^{\mathbf{2}}\right) \\
\mathbf{I} & -\mathbf{K}_{\mathbf{x}}
\end{array}\right]\left[\begin{array}{c}
\dot{\mathbf{E}}_{\mathbf{x}} \\
\mathbf{E}_{\mathbf{x}}
\end{array}\right] .
$$

Here $\mathbf{K}_{\mathbf{x}, \mathbf{0}}$ is a $2 N \times 2 N$ diagonal matrix with elements corresponding to the phase difference between the boundaries of the unit cell of each eigenmode, and $\mathbf{K}_{\mathbf{x}}$ is an $N \times N$ diagonal matrix with elements $k_{x, m}=m K$. Equation (5) can be identified as an eigenvalue equation from which the matrix of phase constants $\mathbf{K}_{\mathbf{x}, \mathbf{0}}$ can be obtained.

\section{SIMULATION RESULTS}

\section{A. Absorption spectrum of a semi-infinite metallic layer with sinusoidal modulation of $\omega_{p}$}

As a first application of the modified FMM, we first consider the following toy geometry: A TM plane wave is normally incident upon a semi-infinite metallic layer, with modulation of the plasma frequency given by $\omega_{p}^{2}=\omega_{p, 0}^{2}+\omega_{p, 1}^{2} \cos \left(\frac{2 \pi x}{L}\right)$. The material parameters are $\gamma=\omega_{p, 0} / 300, v_{F}=0.01 c$, and $\lambda_{p} \equiv \frac{2 \pi c}{\omega_{p, 0}}=5 L$. The calculation has been repeated for the following three modulation amplitudes: $\omega_{p, 1}^{2}=0.1 \omega_{p, 0}^{2}$, $0.05 \omega_{p, 0}^{2}$, and 0 . The results are shown in Fig. 2(a). Two sets of absorption peaks are observed: (1) Absorption peaks near the frequencies $\sim(1 / \sqrt{2}) \omega_{p, 0}$-these are surface waves [surface-plasmon polariton (SPP) like] that are confined near the interface. (2) Absorption oscillations appearing near $\omega_{p, 0}$, which are the consequence of longitudinal waves-it is seen
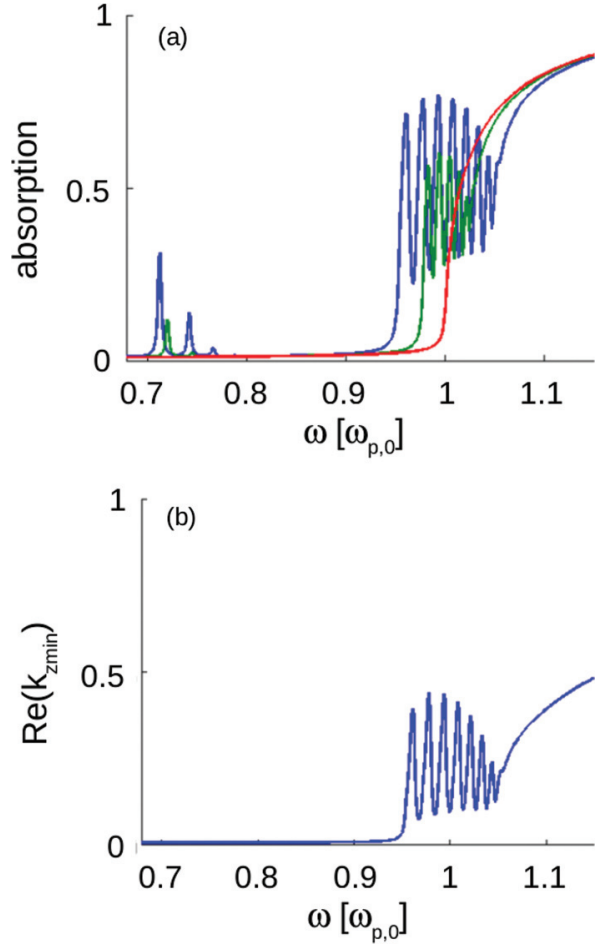

FIG. 2. (Color online) (a) Absorption spectrum of a semi-infinite metallic layer with $\lambda_{p}=\frac{2 \pi c}{\omega_{p, 0}}=5 L$. Blue line: $\omega_{p, 1}^{2}=0.1 \omega_{p, 0}^{2}$. Green line: $\omega_{p, 1}^{2}=0.05 \omega_{p, 0}^{2}$. Red line: $\omega_{p, 1}^{2}=0$. (b) Real part of the propagation constant with an absolute value closest to zero, calculated for the case where $\omega_{p, 1}^{2}=0.1 \omega_{p, 0}^{2}$.

that the oscillation strength increases as the plasma frequency modulation amplitude increases. In Figs. 3(a) and 3(b) we plot $\nabla \cdot J$ for the two lowest frequency absorption peaks $\left(\frac{\omega}{\omega_{p, 0}}=0.9605\right.$ and 0.9772), and in Figs. 3(c) and 3(d) we plot the same quantities for the first two absorption dips $\left(\frac{\omega}{\omega_{p, 0}}=0.9674\right.$ and 0.9841$)$. Since $\nabla \cdot J$ is proportional to the induced charge density [see Eq. (1d)], it can be seen that for the lower frequency modes, the induced charge density concentrates in the middle of the unit cell where the plasma frequency is minimal. This is consistent with previous studies where a layer of metal with lower plasma frequency was deposited on top of a higher plasma frequency metal. For such a case, standing waves in the lower plasma frequency region, similar to those in a $1 \mathrm{D}$ potential well, were observed (see Ref. 3, Sec. 3.4). In addition, the calculation of $H_{y}$ which is a transverse field quantity (no magnetic field exists in a longitudinal mode) shows that the magnetic field is negligible compared to $\nabla \cdot J$, manifesting that the modes are almost completely longitudinal in nature. To reveal the reason for the existence of the absorption peaks and dips, we plot in Fig. 2(b) the real part of the propagation constant with the absolute value closest to zero as a function of frequency, for the case where $\omega_{p, 1}^{2}=0.1 \omega_{p, 0}^{2}$. More mathematically stated, we define $k_{z, \min } \equiv \min \left(\left|k_{z, n}\right|^{2}\right)$ and plot $\operatorname{Re}\left(k_{z, \min }\right)$. There is a clear correspondence between the blue line in Figs. 2(a) and 2(b). The dips and peaks of the absorption spectrum are located at the minima and the maxima of $\operatorname{Re}\left(k_{z, \min }\right)$, respectively. The reason for this is that when $\operatorname{Re}\left(k_{z}\right)$ is relatively large, the longitudinal 

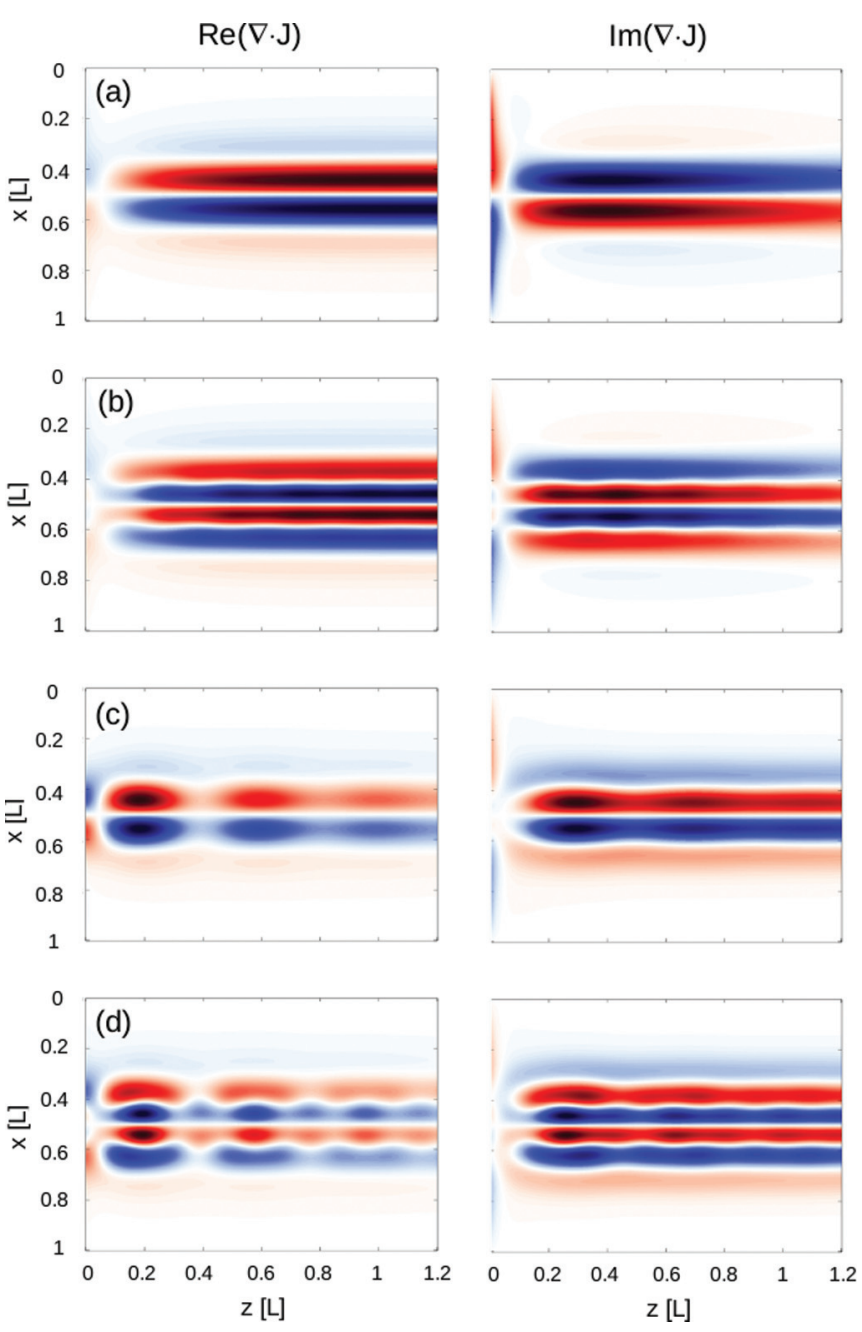

FIG. 3. (Color online) (a), (b) Real and imaginary parts of $\nabla \cdot J$, calculated at the absorption peaks, located at (a) $\frac{\omega}{\omega_{p, 0}}=0.9605$ and (b) $\frac{\omega}{\omega_{p, 0}}=0.9772$. (c), (d) Real and imaginary parts of $\nabla \cdot J$, calculated at the absorption dips, located at (c) $\frac{\omega}{\omega_{p, 0}}=0.9674$ and (d) $\frac{\omega}{\omega_{p, 0}}=$ 0.9841. The air/metal interface is at $z=0$.

mode propagates with significant phase accumulation along the $z$ axis and eventually dissipates. On the other hand, when $\operatorname{Re}\left(k_{z}\right) \cong 0$, the longitudinal mode barely propagates into the metal, but rather has a standing wave pattern along the $x$ axis (see the Supplemental Material ${ }^{31}$ ). This analysis shows the strength of the FMM approach. Being a semianalytical method, it provides physical insight due to the calculation of modes and propagation constants.

\section{B. Absorption spectrum of an $\mathrm{Au} / \mathrm{Ag}$ bimetallic semi-infinite layer}

We now turn to analyze the case of an $\mathrm{Au} / \mathrm{Ag}$ semi-infinite layer. We assume $\omega_{p, \mathrm{Au}}=8.55 \mathrm{eV}, \omega_{p, \mathrm{Ag}}=$ $9.6 \mathrm{eV}, \gamma_{\mathrm{Au}}=\gamma_{\mathrm{Ag}}=0.02 \mathrm{eV}$, and $v_{F, \mathrm{Au}}=v_{F, \mathrm{Ag}}=0.0047 c$. These parameters are from Ref. 32, with the simplifying assumption that the damping in $\mathrm{Au}$ and $\mathrm{Ag}$ is the same. In the unit cell $0 \leqslant x<L$, the plasma frequency is described by $\omega_{p}^{2}(x)=\arctan [(x-0.5 L)(f / L)]\left(\omega_{p, \mathrm{Au}}^{2}-\right.$ $\left.\omega_{p, \mathrm{Ag}}^{2}\right) / \pi+\left(\omega_{p, \mathrm{Au}}^{2}+\omega_{p, \mathrm{Ag}}^{2}\right) / 2$. This function results in a
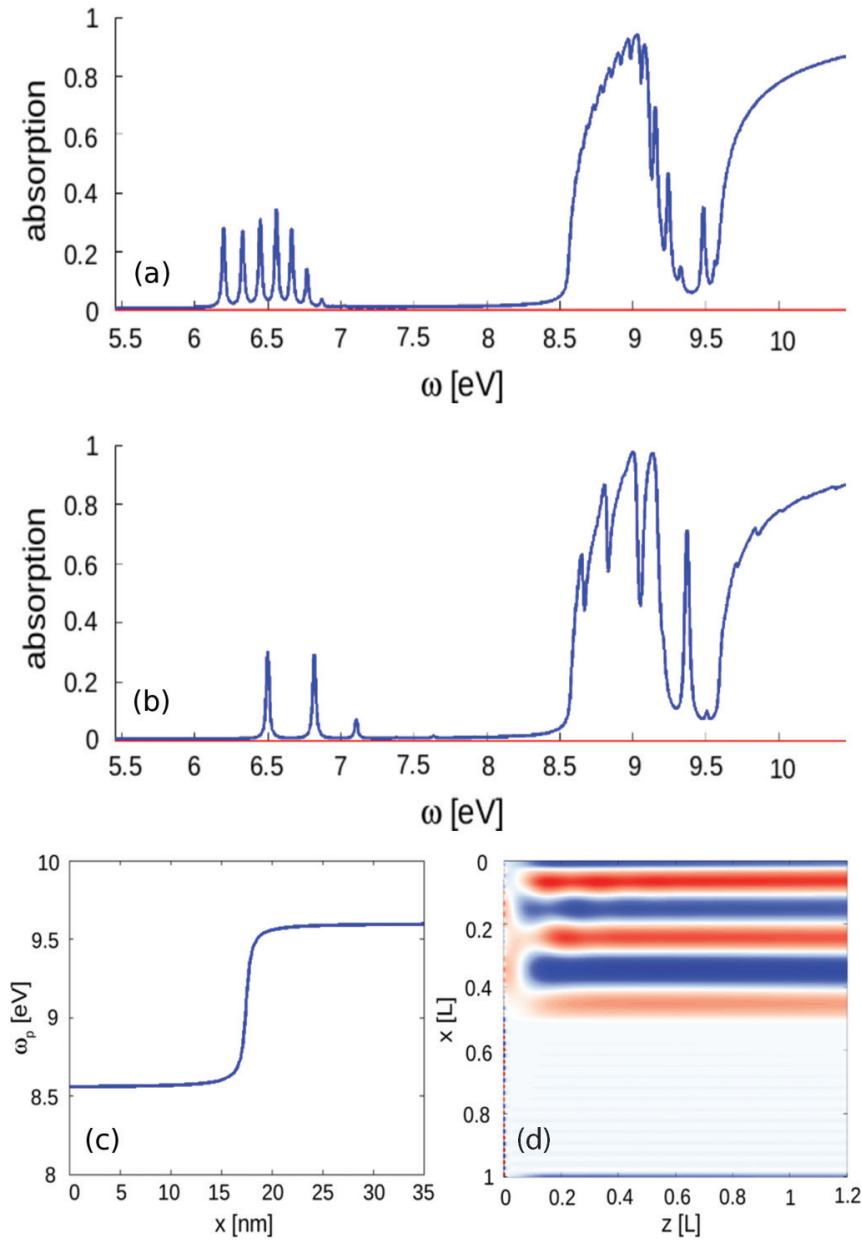

FIG. 4. (Color online) (a) Absorption spectrum of an $\mathrm{Ag} / \mathrm{Au}$ grating with $L=35 \mathrm{~nm}$ as a function of $\omega$. (b) Absorption spectrum of an $\mathrm{Ag} / \mathrm{Au}$ grating with $L=10 \mathrm{~nm}$ as a function of $\omega$. (c) The distribution of $\omega_{p}$ in the unit cell assumed for the calculation. (d) The real part of $\nabla \cdot J$, calculated for $\omega=8.592 \mathrm{eV}$ and $L=35 \mathrm{~nm}$.

continuous steplike profile. The advantage of using such a function is that it eliminates the need to take care of the correct factorization rules of a piecewise discontinuous function, ${ }^{21,33}$ and also provides a more realistic description of the transition between the two metals. The parameter $f$ determines the steepness of the transition between both media. In Fig. 4(a) we plot the absorption spectrum, for $f=100$ and $L=35 \mathrm{~nm}$. The steplike distribution of $\omega_{p}$ in the unit cell is shown in Fig. 4(c). Similarly to the case studied in Sec. III A, the
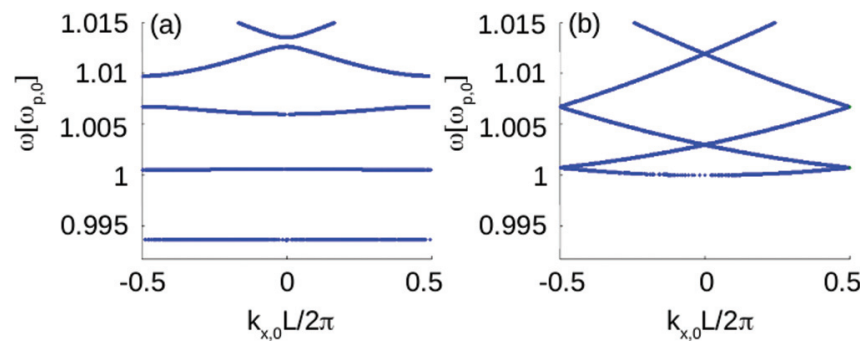

FIG. 5. (Color online) Dispersion of the longitudinal modes. (a) Periodic case with $\omega_{p, 1}^{2}=0.02 \omega_{p, 0}^{2}$. (b) Uniform case $\left(\omega_{p, 1}^{2}=0\right)$. 

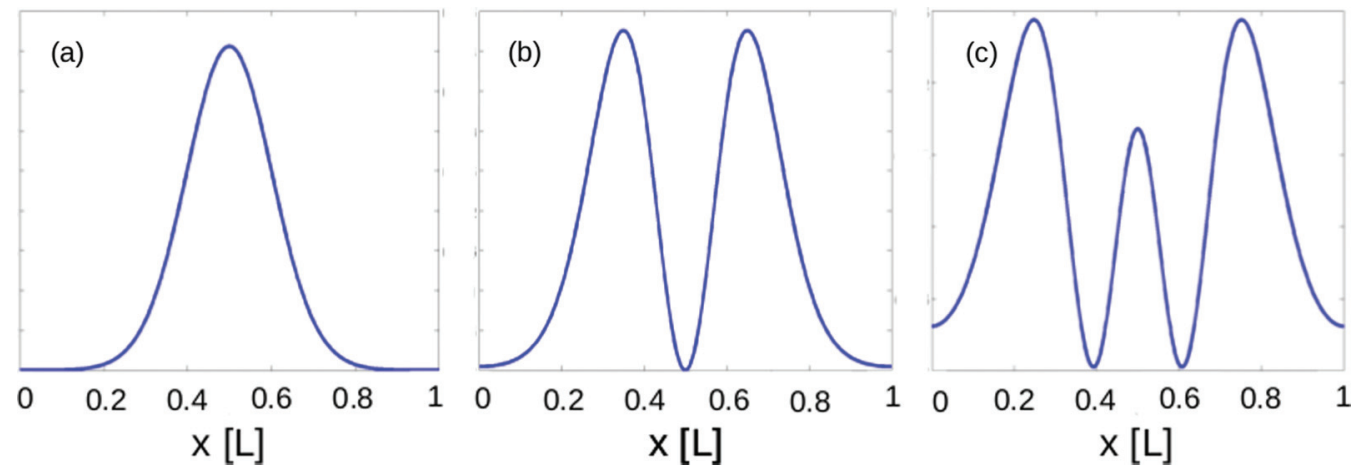

FIG. 6. (Color online) The profile of $\left|J_{x}\right|^{2}$ for the first to third bands of the dispersion diagram plotted in Fig. 5(a). (a) $\omega=0.9936 \omega_{p, 0}$, (b) $\omega=1.0005 \omega_{p, 0}$, (c) $\omega=1.0064 \omega_{p, 0}$. All modes are calculated for $k_{x, 0}=0$.

absorption spectrum exhibts peaks near $(1 / \sqrt{2}) \omega_{p, \text { Au }}$ and $(1 / \sqrt{2}) \omega_{p, \mathrm{Ag}}$. Additionally, there are absorption peaks due to longitudinal modes for $\omega_{p, \mathrm{Au}}<\omega<\omega_{p, \mathrm{Ag}}$. The reason no absorption peaks are observed for $\omega>\omega_{p, \mathrm{Ag}}$ is that the unit cell is larger than the typical dimension $(\sim 10 \mathrm{~nm})$ for which nonlocal effects are significant for these metals. However, when $\omega_{p, \mathrm{Au}}<\omega<\omega_{p, \mathrm{Ag}}$, longitudinal modes exist only in the Au layer, which, for the assumed unit cell size, is small enough to clearly observe longitudinal resonances. Indeed, for a smaller unit cell with $L=10 \mathrm{~nm}$, resonances of longitudinal modes for $\omega>\omega_{p, \mathrm{Ag}}$ are observed [see Fig. 4(b)]. In Fig. 4(d), we show $\nabla \cdot J$ calculated for $\omega=8.592 \mathrm{eV}$ and $L=35 \mathrm{~nm}$. It can be observed that the longitudinal modes are confined in the Au layer only.

\section{Band diagram calculation}

We now turn to calculating the 1D dispersion diagram of longitudinal modes, based on the formulation described in Sec. II B. We assume the following parameters: $\gamma=0$ (no losses), $v_{F}=0.01 c$, and $\lambda_{p}=\frac{2 \pi c}{\omega_{p, 0}}=10 L$.

In Fig. 5(a) we assumed $\omega_{p, 1}^{2}=0.02 \omega_{p, 0}^{2}$. For this case both the band gaps at the edges of the first Brillouin zone (BZ) and at $k_{x, 0}=0$ are apparent. Moreover, the dispersion of the lower order modes is flat, which is an indication for a very low group velocity, regardless of the specific momentum value. This is in contrast to the more conventional case of a periodic structure which generates slow light only at the edges of the BZ. Losses (neglected here for simplicity), however, cause broadening and enhance the group velocity at the band edges. ${ }^{34}$ In Fig. 5(b) we assumed a uniform metallic medium having no modulation, i.e., $\omega_{p, 1}^{2}=0$. Obviously, for this case, no band gaps are observed, as expected.

In Fig. 6 we plot the mode profile of $\left|J_{x}(x)\right|^{2}$ (which is proportional to the kinetic energy of the charges) for the first three bands of the dispersion diagram plotted in Fig. 5(a). As discussed before, it is seen that the lowest energy mode is concentrated in the region of smaller plasma frequency.

\section{CONCLUSION}

In summary, we have developed a semianalytical modal approach to solve Maxwell's equations in the presence of the linearized hydrodynamic correction. With this approach, the diffraction from a periodic metallic layer was calculated. The modal method was shown to provide physical insight into the calculated absorption spectrum, by detailed inspection of the modal propagation constants. Longitudinal modes with propagation constants close to zero were found to generate absorption dips, while modes with maximal propagation constants were related to the absorption peaks. Moreover, we presented a general boundary condition matching scheme, based on the $S$-matrix algorithm, that incorporated the ABC needed to match between local and nonlocal media. In addition, a variant of the PWM was formulated and used in order to calculate the band diagram dispersion of the longitudinal modes. These numerical tools might provide a useful framework for the design of plasmonic circuit devices at the very deep nanoscale. ${ }^{35}$

\section{ACKNOWLEDGMENTS}

This research was supported by the AFOSR. A.Y. acknowledges the support of the CAMBR and Brojde fellowships.

\section{APPENDIX A: DERIVATION OF THE EIGENVALUE EQUATION}

In this Appendix we outline the procedure of derivation of Eq. (2) from Eq. (1). Equations (1a)-(1c) are rewritten as

$$
\begin{aligned}
\frac{\partial}{\partial z} E_{x}= & \frac{\partial}{\partial x} E_{z}+j \omega \mu_{0} H_{y}, \\
\frac{\partial}{\partial z} H_{y}= & +j \omega \varepsilon_{0} E_{x}-j m^{-1} \omega \hat{\omega}^{-2} N_{0}(x) e^{2} E_{x} \\
& -\hat{\omega}^{-2} \beta^{2} \frac{\partial}{\partial x} \nabla \cdot J, \\
\frac{\partial}{\partial z} E_{z}= & -\frac{\partial}{\partial x} E_{x}-\left(i \omega \varepsilon_{0}\right)^{-1} \nabla \cdot J .
\end{aligned}
$$

Likewise, Eqs. (1d) and (1e) are combined to give

$$
\begin{aligned}
\frac{\partial}{\partial z} \nabla \cdot J= & \beta^{-2} \hat{\omega}^{2}\left(\frac{\partial}{\partial x} H_{y}+j \omega \varepsilon_{0} E_{z}\right) \\
& -j \omega N_{0}(x) e^{2} \beta^{-2} m^{-1} E_{z},
\end{aligned}
$$


where $J_{x}$ and $J_{z}$ are eliminated from Eqs. (A1b) and (A1d) according to

$$
\begin{aligned}
& J_{z}=-\frac{\partial}{\partial x} H_{y}-j \omega \varepsilon_{0} E_{z}, \\
& J_{x}=-j m^{-1} \omega \hat{\omega}^{-2} N_{0}(x) e^{2} E_{x}-\hat{\omega}^{-2} \beta^{2} \frac{\partial}{\partial x} \nabla \cdot J .
\end{aligned}
$$

Using the parameters defined in Sec. II, Eq. (A1) can be written as

$$
\begin{aligned}
k_{z} E_{x} & =k_{x} E_{z}+\tilde{\mu}_{0} H_{y}, \\
k_{z} H_{y} & =\tilde{\varepsilon}_{0} E_{x}-\tilde{\varepsilon}_{0} \hat{k}_{0}^{-2} \tilde{\omega}_{p}^{2}(x) E_{x}-\hat{k}_{0}^{-2} \tilde{\beta}^{2} k_{x} \nabla \cdot J, \\
k_{z} E_{z} & =\tilde{\mu}_{0} k_{0}^{-2} \nabla \cdot J-k_{x} E_{x}, \\
k_{z} \nabla \cdot J & =\tilde{\beta}^{-2} \hat{k}_{0}^{2} k_{x} H_{y}+\tilde{\varepsilon}_{0} \tilde{\beta}^{-2} \hat{k}_{0}^{2} E_{z}-\tilde{\varepsilon}_{0} \tilde{\omega}_{p}^{2}(x) \tilde{\beta}^{-2} E_{z} .
\end{aligned}
$$

Equations (A3) can be written in matrix form, as two first-order coupled differential equations:

$$
\begin{gathered}
{\left[\begin{array}{c}
\mathbf{E}_{\mathbf{x}} \\
\nabla \cdot \mathbf{J}
\end{array}\right]\left[\mathbf{K}_{\mathbf{z}}\right]=\left[\begin{array}{cc}
\tilde{\mu}_{0} \mathbf{I} & \mathbf{K}_{\mathbf{x}} \\
\tilde{\beta}^{-2} \hat{k}_{0}^{2} \mathbf{K}_{\mathbf{x}} & \tilde{\varepsilon}_{0} \tilde{\beta}^{-2}\left(\hat{k}_{0}^{2} \mathbf{I}-\Omega_{\mathbf{p}}^{2}\right)
\end{array}\right]\left[\begin{array}{c}
\mathbf{H}_{\mathbf{y}} \\
\mathbf{E}_{\mathbf{z}}
\end{array}\right],} \\
{\left[\begin{array}{c}
\mathbf{H}_{\mathbf{y}} \\
\mathbf{E}_{\mathbf{z}}
\end{array}\right]\left[\mathbf{K}_{\mathbf{z}}\right]=\left[\begin{array}{cc}
\tilde{\varepsilon}_{0}\left(\mathbf{I}-\hat{k}_{0}^{-2} \Omega_{\mathbf{p}}^{2}\right) & -\hat{k}_{0}^{-2} \tilde{\beta}^{2} \mathbf{K}_{\mathbf{x}} \\
-\mathbf{K}_{\mathbf{x}} & \tilde{\mu}_{0} k_{0}^{-2} \mathbf{I}
\end{array}\right]\left[\begin{array}{c}
\mathbf{E}_{\mathbf{x}} \\
\nabla \cdot \mathbf{J}
\end{array}\right] .}
\end{gathered}
$$

By eliminating $\mathbf{H}_{\mathbf{y}}$ and $\mathbf{E}_{\mathbf{z}}$, Eqs. (A4a) and (A4b) are combined to obtain Eq. (2).

\section{APPENDIX B: $S$-MATRIX FORMULATION FOR NONLOCAL PERIODIC LAYERS EMBEDDED IN A LOCAL ENVIRONMENT}

In this Appendix, the $S$ matrix for a periodic layer with nonlocal response, embedded in a local environment, is evaluated. The geometry is defined in Fig. 7. Two local layers, labeled as " $L_{1}$ " and " $L_{2}$," surround a nonlocal slab labeled as "NL." The mode amplitude constants are defined as $C$, with the subscript denoting the layer index, and the superscript the propagation direction, with "+" and "-" standing for waves propagating in the positive and negative $z$ directions, respectively. Each vector of mode amplitudes

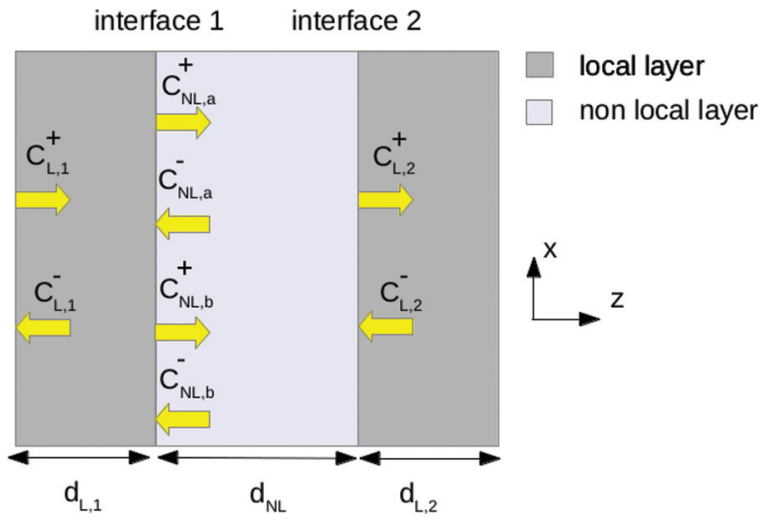

FIG. 7. (Color online) Schematic showing a nonlocal layer embedded in a local environment.
$C$ has $N$ elements. The nonlocal layer therefore supports twice as many modes as the local layers, consistent with the discussion in Sec. II. For the case where the nonlocal layer is nonperiodic, the solution of Eq. (2) results in $N$ pure transverse and $N$ pure longitudinal modes. Then, the mode amplitude constants can be divided into two groups, $C_{\mathrm{NL} \text {, a }}$ and $C_{\mathrm{NL}, \mathrm{b}}$, with each of these groups associated with either longitudinal or transverse modes. When the nonlocal layer is periodic, no pure longitudinal modes exist in the general case. For consistency, we keep the division into two groups $C_{\mathrm{NL}, \mathrm{a}}$ and $C_{\mathrm{NL}, \mathrm{b}}$ so that each mode amplitude vector remains with $N$ elements. However, the division into these two groups is now arbitrary. To find the mode amplitude vectors, we employ the $S$-matrix approach, for which a scattering matrix $\mathbf{S}$ relates a vector of incident mode amplitudes a to a vector of outgoing mode amplitudes $\mathbf{b}$ according to $\mathbf{b}=\mathbf{S a}$. This approach is considered as numerically stable in the sense that growing exponential terms are avoided. ${ }^{22}$ First, the $S$ matrix for the interfaces are derived and afterwards they are used to derive the layer $S$ matrix.

The two interface $S$ matrices are defined as

$$
\left[\begin{array}{ccc}
S_{11}^{(1)} & S_{12}^{(1)} & S_{13}^{(1)} \\
S_{21}^{(1)} & S_{22}^{(1)} & S_{23}^{(1)} \\
S_{31}^{(1)} & S_{32}^{(1)} & S_{33}^{(1)}
\end{array}\right]\left[\begin{array}{c}
P_{\mathrm{L}, 1}^{+} C_{\mathrm{L}, 1}^{+} \\
C_{\mathrm{NL}, \mathrm{a}}^{-} \\
C_{\mathrm{NL}, \mathrm{b}}^{-}
\end{array}\right]=\left[\begin{array}{c}
C_{\mathrm{NL}, \mathrm{a}}^{+} \\
C_{\mathrm{N}, \mathrm{b}}^{+} \\
P_{\mathrm{L}, 1}^{-} C_{\mathrm{L}, 1}^{-}
\end{array}\right],
$$

$$
\left[\begin{array}{ccc}
S_{11}^{(2)} & S_{12}^{(2)} & S_{13}^{(2)} \\
S_{21}^{(2)} & S_{22}^{(2)} & S_{23}^{(2)} \\
S_{31}^{(2)} & S_{32}^{(2)} & S_{33}^{(2)}
\end{array}\right]\left[\begin{array}{c}
C_{\mathrm{L}, 2}^{-} \\
P_{\mathrm{NL}, \mathrm{a}}^{+} C_{\mathrm{NL}, \mathrm{a}}^{+} \\
P_{\mathrm{NL}, \mathrm{b}}^{+} C_{\mathrm{N}, \mathrm{b}}^{+}
\end{array}\right]=\left[\begin{array}{c}
P_{\mathrm{NL}, \mathrm{a}}^{-} C_{\mathrm{NL}, \mathrm{a}}^{-} \\
P_{\mathrm{NL}, \mathrm{b}}^{-} C_{\mathrm{NL}, \mathrm{b}} \\
C_{\mathrm{L}, 2}^{+}
\end{array}\right] .
$$

Here, the phase matrices $P_{\mathrm{L}, 1}^{ \pm}$are $N \times N$ diagonal matrices with elements $\exp \left(j k_{0} k_{z, n}^{(i) \pm} d_{\mathrm{L}, 1}\right)$, with $d_{\mathrm{L}, 1}$ the local layer thickness as shown in Fig. 7. The forward and backward propagating modes in layer $i$ have propagation constants $k_{z, n}^{(i)+}$ and $k_{z, n}^{(i)-}$, respectively. Since $k_{z, n}^{(i)+}=-k_{z, n}^{(i)-}$, these phase matrices satisfy $P_{\mathrm{L}, \mathrm{i}}^{+}=\left(P_{\mathrm{L}, \mathrm{i}}^{-}\right)^{-1}$. For the nonlocal layer the phase matrices are $P_{\mathrm{NL}, \mathrm{x}}^{ \pm}$with $x=a, b$, with the subscripts and superscripts having their obvious meaning. The phase matrices appear in Eq. (B1) because the mode amplitudes are defined to have zero phase at the left boundary of each layer.

Assuming continuity of $E_{x}, J_{z}$, and $E_{z}$, we match the eigenvector matrices of these field quantities at the first interface:

$$
\begin{aligned}
{\left[\begin{array}{cc}
E_{x, \mathrm{~L}, 1}^{+} & E_{x, \mathrm{~L}, 1}^{-} \\
J_{z, \mathrm{~L}, 1}^{+} & J_{z, \mathrm{~L}, 1}^{-} \\
E_{z, \mathrm{~L}, 1}^{+} & E_{z, \mathrm{~L}, 1}^{-}
\end{array}\right]\left[\begin{array}{c}
P_{\mathrm{L}, 1}^{+} C_{\mathrm{L}, 1}^{+} \\
P_{\mathrm{L}, 1}^{-} C_{\mathrm{L}, 1}^{-}
\end{array}\right] } \\
=\left[\begin{array}{cccc}
E_{x, \mathrm{NL}, \mathrm{a}}^{+} & E_{x, \mathrm{NL}, \mathrm{b}}^{+} & E_{x, \mathrm{NL}, \mathrm{a}}^{-} & E_{x, \mathrm{NL}, \mathrm{b}}^{-} \\
J_{z, \mathrm{NL}, \mathrm{a}}^{+} & J_{z, \mathrm{NL}, \mathrm{b}}^{+} & J_{z, \mathrm{NL}, \mathrm{a}}^{-} & J_{z, \mathrm{NL}, \mathrm{b}}^{-} \\
E_{z, \mathrm{NL}, \mathrm{a}}^{+} & E_{z, \mathrm{NL}, \mathrm{b}}^{+} & E_{z, \mathrm{NL}, \mathrm{a}}^{-} & E_{z, \mathrm{NL}, \mathrm{b}}^{-}
\end{array}\right]\left[\begin{array}{c}
C_{\mathrm{NL}, \mathrm{a}}^{+} \\
C_{\mathrm{NL}, \mathrm{b}}^{+} \\
C_{\mathrm{NL}, \mathrm{a}}^{-} \\
C_{\mathrm{NL}, \mathrm{b}}^{-}
\end{array}\right],
\end{aligned}
$$

where a "+" or "-" superscript of the eigenvector matrices represents left and right propagating field quantities, respectively. 
Rearranging terms in Eq. (B2) we obtain

$$
\left[\begin{array}{ccc}
E_{x, \mathrm{~L}, 1}^{+} & -E_{x, \mathrm{NL}, \mathrm{a}}^{-} & -E_{x, \mathrm{NL}, \mathrm{b}}^{-} \\
J_{z, \mathrm{~L}, 1}^{+} & -J_{z, \mathrm{NL}, \mathrm{a}}^{-} & -J_{z, \mathrm{NL}, \mathrm{b}}^{-} \\
E_{z, \mathrm{~L}, 1}^{+} & -E_{z, \mathrm{NL}, \mathrm{a}}^{-} & -E_{z, \mathrm{NL}, \mathrm{b}}^{-}
\end{array}\right]\left[\begin{array}{c}
P_{\mathrm{L}, 1}^{+} C_{\mathrm{L}, 1}^{+} \\
C_{\mathrm{NL}, \mathrm{a}}^{-} \\
C_{\mathrm{NL}, \mathrm{b}}^{-}
\end{array}\right]=\left[\begin{array}{ccc}
E_{x, \mathrm{NL}, \mathrm{a}}^{+} & E_{x, \mathrm{NL}, \mathrm{b}}^{+} & -E_{x, \mathrm{~L}, 1}^{-} \\
J_{z, \mathrm{NL}, \mathrm{a}}^{+} & J_{z, \mathrm{NL}, \mathrm{b}}^{+} & -J_{z, \mathrm{~L}, 1}^{-} \\
E_{z, \mathrm{NL}, \mathrm{a}}^{+} & E_{z, \mathrm{NL}, \mathrm{b}}^{+} & -E_{z, \mathrm{~L}, 1}^{-}
\end{array}\right]\left[\begin{array}{c}
C_{\mathrm{NL}, \mathrm{a}}^{+} \\
C_{\mathrm{NL}, \mathrm{b}}^{+} \\
P_{\mathrm{L}, 1}^{-} C_{\mathrm{L}, 1}^{-}
\end{array}\right] .
$$

Comparing Eq. (B3) with (B1a), it is seen that the first interface $S$ matrix can be expressed by the eigenvector matrices as

$$
\left[\begin{array}{ccc}
S_{11}^{(1)} & S_{12}^{(1)} & S_{13}^{(1)} \\
S_{21}^{(1)} & S_{22}^{(1)} & S_{23}^{(1)} \\
S_{31}^{(1)} & S_{32}^{(1)} & S_{33}^{(1)}
\end{array}\right]=\left[\begin{array}{ccc}
E_{x, \mathrm{NL}, \mathrm{a}}^{+} & E_{x, \mathrm{NL}, \mathrm{b}}^{+} & -E_{x, \mathrm{~L}, 1}^{-} \\
J_{z, \mathrm{NL}, \mathrm{a}}^{+} & J_{z, \mathrm{NL}, \mathrm{b}}^{+} & -J_{z, \mathrm{~L}, 1}^{-} \\
E_{z, \mathrm{NL}, \mathrm{a}}^{+} & E_{z, \mathrm{NL}, \mathrm{b}}^{+} & -E_{z, \mathrm{~L}, 1}^{-}
\end{array}\right]^{-1}\left[\begin{array}{ccc}
E_{x, \mathrm{~L}, 1}^{+} & -E_{x, \mathrm{NL}, \mathrm{a}}^{-} & -E_{x, \mathrm{NL}, \mathrm{b}}^{-} \\
J_{z, \mathrm{~L}, 1}^{+} & -J_{z, \mathrm{NL}, \mathrm{a}}^{-} & -J_{z, \mathrm{NL}, \mathrm{b}}^{-} \\
E_{z, \mathrm{~L}, 1}^{+} & -E_{z, \mathrm{NL}, \mathrm{a}}^{-} & -E_{z, \mathrm{NL}, \mathrm{b}}^{-}
\end{array}\right] .
$$

Following a similar procedure, the second interface $S$ matrix is

$$
\left[\begin{array}{ccc}
S_{11}^{(2)} & S_{12}^{(2)} & S_{13}^{(2)} \\
S_{21}^{(2)} & S_{22}^{(2)} & S_{23}^{(2)} \\
S_{31}^{(2)} & S_{32}^{(2)} & S_{33}^{(2)}
\end{array}\right]=\left[\begin{array}{ccc}
E_{x, \mathrm{NL}, \mathrm{a}}^{-} & E_{x, \mathrm{NL}, \mathrm{b}}^{-} & -E_{x, \mathrm{~L}, 2}^{+} \\
J_{z, \mathrm{NL}, \mathrm{a}}^{-} & J_{z, \mathrm{NL}, \mathrm{b}}^{-} & -J_{z, \mathrm{~L}, 2}^{+} \\
E_{z, \mathrm{NL}, \mathrm{a}}^{-} & E_{z, \mathrm{NL}, \mathrm{b}}^{-} & -E_{z, \mathrm{~L}, 2}^{+}
\end{array}\right]^{-1}\left[\begin{array}{ccc}
E_{x, \mathrm{~L}, 2}^{-} & -E_{x, \mathrm{NL}, \mathrm{a}}^{+} & -E_{x, \mathrm{NL}, \mathrm{b}}^{+} \\
J_{z, \mathrm{~L}, 2}^{-} & -J_{z, \mathrm{NL}, \mathrm{a}}^{+} & -J_{z, \mathrm{NL}, \mathrm{b}}^{+} \\
E_{z, \mathrm{~L}, 2}^{-} & -E_{z, \mathrm{NL}, \mathrm{a}}^{+} & -E_{z, \mathrm{NL}, \mathrm{b}}^{+}
\end{array}\right] .
$$

By detaching the phase matrices, Eq. (B1) can be modified to

$$
\begin{aligned}
& {\left[\begin{array}{ccc}
I & 0 & 0 \\
0 & I & 0 \\
0 & 0 & \left(P_{\mathrm{L}, 1}^{-}\right)^{-1}
\end{array}\right]\left[\begin{array}{ccc}
S_{11}^{(1)} & S_{12}^{(1)} & S_{13}^{(1)} \\
S_{21}^{(1)} & S_{22}^{(1)} & S_{23}^{(1)} \\
S_{31}^{(1)} & S_{32}^{(1)} & S_{33}^{(1)}
\end{array}\right]\left[\begin{array}{ccc}
P_{\mathrm{L}, 1}^{+} & 0 & 0 \\
0 & I & 0 \\
0 & 0 & I
\end{array}\right]\left[\begin{array}{c}
C_{\mathrm{L}, 1}^{+} \\
C_{\mathrm{NL}, \mathrm{a}}^{-} \\
C_{\mathrm{NL}, \mathrm{b}}^{-}
\end{array}\right]=\left[\begin{array}{c}
C_{\mathrm{NL}, \mathrm{a}}^{+} \\
C_{\mathrm{NL}, \mathrm{b}}^{+} \\
C_{\mathrm{L}, 1}^{-}
\end{array}\right],} \\
& {\left[\begin{array}{ccc}
\left(P_{\mathrm{NL}, \mathrm{a}}^{-}\right)^{-1} & 0 & 0 \\
0 & \left(P_{\mathrm{NL}, \mathrm{b}}^{-}\right)^{-1} & 0 \\
0 & 0 & I
\end{array}\right]\left[\begin{array}{ccc}
S_{11}^{(2)} & S_{12}^{(2)} & S_{13}^{(2)} \\
S_{21}^{(2)} & S_{22}^{(2)} & S_{23}^{(2)} \\
S_{31}^{(2)} & S_{32}^{(2)} & S_{33}^{(2)}
\end{array}\right]\left[\begin{array}{ccc}
I & 0 & 0 \\
0 & P_{\mathrm{NL}, \mathrm{a}}^{+} & 0 \\
0 & 0 & P_{\mathrm{NL}, \mathrm{b}}^{+}
\end{array}\right]\left[\begin{array}{c}
C_{\mathrm{L}, 2}^{-} \\
C_{\mathrm{NL}, \mathrm{a}}^{+} \\
C_{\mathrm{NL}, \mathrm{b}}^{+}
\end{array}\right]=\left[\begin{array}{c}
C_{\mathrm{NL}, \mathrm{a}}^{-} \\
C_{\mathrm{NL}, \mathrm{b}} \\
C_{\mathrm{L}, 2}^{+}
\end{array}\right] .}
\end{aligned}
$$

Performing the matrix multiplications in Eq. (B5) and rearranging terms, we obtain two homogeneous equations:

$$
\begin{gathered}
{\left[\begin{array}{cccccc}
S_{11}^{(1)} P_{\mathrm{L}, 1}^{+} & S_{12}^{(1)} & S_{13}^{(1)} & -I & 0 & 0 \\
S_{21}^{(1)} P_{\mathrm{L}, 1}^{+} & S_{22}^{(1)} & S_{23}^{(1)} & 0 & -I & 0 \\
P_{\mathrm{L}, 1}^{+} S_{31}^{(1)} P_{\mathrm{L}, 1}^{+} & P_{\mathrm{L}, 1}^{+} S_{32}^{(1)} & P_{\mathrm{L}, 1}^{+} S_{33}^{(1)} & 0 & 0 & -I
\end{array}\right]\left[\begin{array}{c}
C_{\mathrm{L}, 1}^{+} \\
C_{\mathrm{NL}, \mathrm{a}}^{+} \\
C_{\mathrm{NL}, \mathrm{b}}^{+} \\
C_{\mathrm{NL}, \mathrm{a}}^{-} \\
C_{\mathrm{NL}, \mathrm{b}}^{-} \\
C_{\mathrm{L}, 1}^{-}
\end{array}\right]=0,} \\
{\left[\begin{array}{ccccccc}
P_{\mathrm{NL}, \mathrm{a}}^{+} S_{11}^{(2)} & P_{\mathrm{NL}, \mathrm{a}}^{+} S_{12}^{(2)} P_{\mathrm{NL}, \mathrm{a}}^{+} & P_{\mathrm{NL}, \mathrm{a}}^{+} S_{13}^{(2)} P_{\mathrm{NL}, \mathrm{b}}^{+} & -I & 0 & 0 \\
P_{\mathrm{N}, \mathrm{b}}^{+} S_{21}^{(2)} & P_{\mathrm{NL}, \mathrm{b}}^{+} S_{22}^{(2)} P_{\mathrm{NL}, \mathrm{a}}^{+} & P_{\mathrm{NL}, \mathrm{b}}^{+} S_{23}^{(2)} P_{\mathrm{NL}, \mathrm{b}}^{+} & 0 & -I & 0 \\
S_{31}^{(2)} & S_{32}^{(2)} P_{\mathrm{NL}, \mathrm{a}}^{+} & S_{33}^{(2)} P_{\mathrm{NL}, \mathrm{b}}^{+} & 0 & 0 & -I
\end{array}\right]\left[\begin{array}{c}
C_{\mathrm{L}, 2}^{-} \\
C_{\mathrm{NL}, \mathrm{a}}^{+} \\
C_{\mathrm{NL}, \mathrm{b}}^{+} \\
C_{\mathrm{NL}, \mathrm{a}}^{-} \\
C_{\mathrm{NL}, \mathrm{b}}^{-} \\
C_{\mathrm{L}, 2}^{+}
\end{array}\right]=0 .}
\end{gathered}
$$

Combining the two equations in (B6), we obtain a system of equations that connect the incident amplitudes $\left(C_{\mathrm{L}, 1}^{+}\right.$and $\left.C_{\mathrm{L}, 2}^{-}\right)$with the outgoing $\left(C_{\mathrm{L}, 2}^{+}\right.$and $\left.C_{\mathrm{L}, 1}^{-}\right)$and internal amplitudes $\left(C_{\mathrm{NL}, \mathrm{a}}^{+}, C_{\mathrm{NL}, \mathrm{b}}^{+}, C_{\mathrm{NL}, \mathrm{a}}^{-}\right.$, and $\left.C_{\mathrm{NL}, \mathrm{b}}^{-}\right)$:

$$
\begin{aligned}
& S_{\text {layer }}\left[\begin{array}{c}
C_{\mathrm{L}, 1}^{+} \\
C_{\mathrm{L}, 2}^{-}
\end{array}\right]= {\left[\begin{array}{c}
C_{\mathrm{L}, 2}^{+} \\
C_{\mathrm{L}, 1}^{-} \\
C_{\mathrm{NL}, \mathrm{a}}^{+} \\
C_{\mathrm{NL}, \mathrm{b}}^{+} \\
C_{\mathrm{NL}, \mathrm{a}}^{-} \\
C_{\mathrm{NL}, \mathrm{b}}^{-}
\end{array}\right], } \\
& S_{\text {layer }} \equiv A^{-1} B,
\end{aligned}
$$




$$
\begin{aligned}
A & =\left[\begin{array}{cccccc}
0 & 0 & I & 0 & -S_{12}^{(1)} & -S_{13}^{(1)} \\
0 & 0 & 0 & I & -S_{22}^{(1)} & -S_{23}^{(1)} \\
0 & I & 0 & 0 & -P_{\mathrm{L}, 1}^{+} S_{32}^{(1)} & -P_{\mathrm{L}, 1}^{+} S_{33}^{(1)} \\
0 & 0 & -P_{\mathrm{NL}, \mathrm{a}}^{+} S_{12}^{(2)} P_{\mathrm{NL}, \mathrm{a}}^{+} & -P_{\mathrm{NL}, \mathrm{a}}^{+} S_{13}^{(2)} P_{\mathrm{NL}, \mathrm{b}}^{+} & I & 0 \\
0 & 0 & -P_{\mathrm{NL}, \mathrm{b}}^{+} S_{22}^{(2)} P_{\mathrm{NL}, \mathrm{a}}^{+} & -P_{\mathrm{NL}, \mathrm{b}}^{+} S_{23}^{(2)} P_{\mathrm{NL}, \mathrm{b}}^{+} & 0 & I \\
I & 0 & -S_{32}^{(2)} P_{\mathrm{NL}, \mathrm{a}}^{+} & -S_{33}^{(2)} P_{\mathrm{NL}, \mathrm{b}}^{+} & 0 & 0
\end{array}\right], \\
B & =\left[\begin{array}{cc}
S_{11}^{(1)} P_{\mathrm{L}, 1}^{+} & 0 \\
S_{21}^{(1)} P_{\mathrm{L}, 1}^{+} & 0 \\
P_{\mathrm{L}, 1}^{+} S_{31}^{(1)} P_{\mathrm{L}, 1}^{+} & 0 \\
0 & P_{\mathrm{NL}, \mathrm{a}}^{+} S_{11}^{(2)} \\
0 & P_{\mathrm{NL}, \mathrm{b}}^{+(2)} \\
0 & S_{31}^{(2)}
\end{array}\right] .
\end{aligned}
$$

$S_{\text {layer }}$ is a $6 N \times 2 N$ matrix, which can be divided into two submatrices according to

$$
\begin{aligned}
S_{\text {layer }} & \equiv\left[\begin{array}{c}
S_{\text {external }} \\
S_{\text {internal }}
\end{array}\right], \\
S_{\text {external }} & \equiv\left[\begin{array}{ll}
S_{\text {layer,11 }} & S_{\text {layer,12 }} \\
S_{\text {layer,21 }} & S_{\text {layer,22 }}
\end{array}\right], \\
S_{\text {internal }} & \equiv\left[\begin{array}{ll}
S_{\text {layer,31 }} & S_{\text {layer,32 }} \\
S_{\text {layer,41 }} & S_{\text {layer,42 }} \\
S_{\text {layer,51 }} & S_{\text {layer,52 }} \\
S_{\text {layer,61 }} & S_{\text {layer,62 }}
\end{array}\right] .
\end{aligned}
$$

Equations (B7) and (B8) provide a complete description of all mode amplitudes. $S_{\text {external }}$ can be regarded as the ordinary $2 N \times 2 N$ $S$ matrix, which couples transverse only modes in the first local layer to transverse only modes in the second local layer. From $S_{\text {internal }}$ the internal (i.e., nonlocal) amplitudes $C_{\mathrm{NL}, \mathrm{a}}^{+}, C_{\mathrm{NL}, \mathrm{b}}^{+}, C_{\mathrm{NL}, \mathrm{a}}^{-}$, and $C_{\mathrm{NL}, \mathrm{b}}^{-}$can be obtained.

*ulevy@mail.huji.ac.il

${ }^{1}$ P. J. Feibelman, Prog. Surf. Sci. 12, 287 (1982).

${ }^{2}$ A. D. Boardman, Electromagnetic Surface Modes (Wiley, New York, 1982).

${ }^{3}$ F. Forstmann and R. R. Gerhardts, Metal Optics Near the Plasma Frequency (Springer, Berlin, 1986).

${ }^{4}$ J. M. Pitarke, V. M. Silkin, E. V. Chulkov, and P. M. Echenique, Rep. Prog. Phys. 70, 1 (2007).

${ }^{5}$ M. Anderegg, B. Feuerbacher, and B. Fitton, Phys. Rev. Lett. 27, 1565 (1971).

${ }^{6}$ C. Ciracì, R. T. Hill, J. J. Mock, Y. Urzhumov, A. I. FernándezDomínguez, S. A. Maier, J. B. Pendry, A. Chilkoti, and D. R. Smith, Science 337, 1072 (2012).

${ }^{7}$ S. Raza, N. Stenger, S. Kadkhodazadeh, S. V. Fischer, N. Kostesha, A.-P. Jauho, A. Burrows, M. Wubs, and N. A. Mortensen, Nanophotonics 2, 131 (2013).

${ }^{8}$ T. V. Teperik, P. Nordlander, J. Aizpurua, and A. G. Borisov, Phys. Rev. Lett. 110, 263901 (2013).

${ }^{9}$ R. Esteban, A. G. Borisov, P. Nordlander, and J. Aizpurua, Nat. Commun. 3, 825 (2012).

${ }^{10}$ L. Stella, P. Zhang, F. J. García-Vidal, A. Rubio, and P. GarcíaGonzález, J. Phys. Chem. C 117, 8941 (2013).

${ }^{11}$ R. Ruppin, Opt. Commun. 190, 205 (2001).
${ }^{12}$ F. J. García de Abajo, J. Phys. Chem. C 112, 17983 (2008).

${ }^{13}$ W. L. Mochán, M. del Castillo-Mussot, and R. G. Barrera, Phys. Rev. B 35, 1088 (1987).

${ }^{14}$ V. Yannopapas, J. Phys.: Condens. Matter 20, 325211 (2008).

${ }^{15}$ S. Raza, G. Toscano, A.-P. Jauho, M. Wubs, and N. A. Mortensen, Phys. Rev. B 84, 121412(R) (2011).

${ }^{16}$ A. I. Fernández-Domínguez, A. Wiener, F. J. García-Vidal, S. A. Maier, and J. B. Pendry, Phys. Rev. Lett. 108, 106802 (2012).

${ }^{17}$ G. Toscano, S. Raza, A.-P. Jauho, N. A. Mortensen, and M. Wubs, Opt. Express 20, 4176 (2012).

${ }^{18}$ G. Toscano, S. Raza, W. Yan, C. Jeppesen, S. Xiao, M. Wubs, A.-P. Jauho, S. I. Bozhevolnyi, and N. A. Mortensen, Nanophotonics 2, 161 (2013).

${ }^{19}$ K. R. Hiremath, L. Zschiedrich, and F. Schmidt, J. Comput. Phys. 231, 5890 (2012).

${ }^{20}$ M. G. Moharam, E. B. Grann, D. A. Pommet, and T. K. Gaylord, J. Opt. Soc. Am. A 12, 1068 (1995).

${ }^{21}$ P. Lalanne and G. Morris, J. Opt. Soc. Am. A 13, 779 (1996).

${ }^{22}$ L. Li, J. Opt. Soc. Am. A 13, 1024 (1996).

${ }^{23} \mathrm{H}$. Liu and P. Lalanne, Nature (London) 452, 728 (2008).

${ }^{24}$ A. D. Boardman and R. Ruppin, Surf. Sci. 112, 153 (1981). 
${ }^{25}$ A. R. Melnyk and M. J. Harrison, Phys. Rev. B 2, 835 (1970).

${ }^{26}$ W. Yan, M. Wubs, and N. A. Mortensen, Phys. Rev. B 86, 205429 (2012).

${ }^{27}$ A. Moreau, C. Ciracì, and D. R. Smith, Phys. Rev. B 87, 045401 (2013).

${ }^{28}$ S. Shi, C. Chen, and D. W. Prather, Appl. Phys. Lett. 86, 043104 (2005).

${ }^{29}$ J. D. Joannopoulos, S. G. Johnson, J. N. Winn, and R. D. Meade, Photonic Crystals: Molding the Flow of Light, 2nd ed. (Princeton University Press, Princeton, NJ, 2008).

${ }^{30}$ A. Raman and S. Fan, Phys. Rev. Lett. 104, 087401 (2010).
${ }^{31}$ See Supplemental Material at http://link.aps.org/supplemental/ 10.1103/PhysRevB.88.205120 for an animation, showing $\nabla \cdot J$ at the same frequencies as those in Fig. 3. This animation manifests visually Fig. 2, namely, the phase accumulation in the $z$ direction is apparent for the absorption peaks.

${ }^{32}$ M. G. Blaber, M. D. Arnold, and M. J. Ford, J. Phys. Chem. C 113, 3041 (2009).

${ }^{33}$ L. Li, J. Opt. Soc. Am. A 13, 1870 (1996).

${ }^{34}$ J. G. Pedersen, S. Xiao, and N. A. Mortensen, Phys. Rev. B 78, 153101 (2008).

${ }^{35}$ N. Engheta, Science 317, 1698 (2007). 